periods of sleeplessness, and of impairment of memory. In April, 1925, he developed a tremor and stiffness of the fingers of the left hand, which seriously interfered with his playing. He underwent a fortnight's course of nicotine treatment, the dose being gradually increased from $1 / 30$ grain to $1 / 10$ grain thrice daily. A further increase to $1 / 5$ grain thrice daily was tried but had to be stopped because it caused vomiting. The patient was discharged much improved; although the mask-like expression was still present the movements of the limbs were much freer and the gait steadier. The patient thought that he would be able to play the 'cello again, and, when seen a month later, stated that he had resumed his work and that he was feeling much better.

A youth, aged 17, had an acute attack of encephalitis lethargica in April, 1924, with diplopia, squint, headache, and delirium, until February, 1925, when all the movements commenced to be klow and required a great effort on the patient's part. When I saw him in May, 1925, he was an obvious case of Parkinsonism, with the typical facies, rigidity of the limbs, and marked retropulsion; he would commence to sway backwards, and in order not to fall would start walking backwards, and continue to do so until arrested by a wall or by falling. He underwent nicotine treatment for three weeks, with increasing doses up to $1 / 10$ grain thrice daily. At the end of this course of treatment he had greatly improved; the back-stepping gait had disappeared, and he walked briskly, swinging his arms alongside of him. The expression in his face had also returned, and he could smile normally.

The tone of the muscles was repeatedly examined before and after the nicotine injections, and more especially the capacity of the limb (brought by a passive movement into a new posture), when released, to remain in thăt new posture. Such a property Sherrington has termed "plasticity" of the musculature, and was measured by him by means of the "shortening and lengthening reaction." Thus, if the leg be flexed at the knee, so lengthening the extensor muscles of the thigh, this position is retained ("lengthening reaction"); if the knee be now extended, so shortening the extensor muscles, the new position is again maintained (" shortening reaction").

The "shortening and lengthening reaction" was found in many cases to be definitely diminished after the nicotine injections, and in some it disappeared entirely after the course of treatment.

A man, aged 39, in January, 1924, had a dizzy attack whilst at work, followed by diplopia and drowsiness. He recovered completely from his illness, and was quite well for seven months. Six weeks before his admission to the hospital he gave up lis work because of his inability to use his tools or to wheel a barrow. He presented a typical Parkinsonian syndrome, with mask-like face, marked stiffness of the muscles of the neck, and mass of lateral rotation of the head when looking sideways. "Shortening and lengthening reaction" of the upper limbs was well marked, and sialorrhoea was present. He received nicotine well marked, and sialorrhoea was present. He received nicotine
treatment for three weeks, the dose ranging between $1 / 30$ alid $1 / 10$ grain thrice daily. At the end of the three weeks the stiffness of the neck muscles had disappeared, and likewise the "shortening and lengthening reaction "of the upper limbs. The sialorrhoea was, howerer, unaltered.

Nicotine had no effect on either the tremors or the sialorrhoca. The persistency of the tremors in some cases masked the improvement of the patient's stiffness, so that no real relief was obtained, and the patients would complain of not feeling better because the tremors had not improved. In one case the phenomenon known under the name of "kinesia paradoxa" was observed. A girl, aged 15, who had been undergoing treatment for a fortnight, was seen one day to run up the stairs as if her fetters had suddenly fallen off, but shortly afterwards relapsed into her Parkinsonian state. Some cases showed a marked improvement one week, only to fall back worse than ever the next. Any improvement of this kind was ascribed to the sudden variations and to the spontaneous recoveries so commonly seen in this disease, and not to any therapeutic effect of nicotine.

In observing the patient's progress allowances were made for improvements which might have been caused by suggestion; leading questions were therefore purposely avoided and objective findings, such as a definite diminution of tone, a return of facial expression; or an improvement of the gait, were taken to be of more importance than subjective ones. All the patients were seen again six months later. The cases which had benefited by nicotine treatment had all relapsed into their previous state, and a case which had not responded to it had died. In one case the improvement lasted a month, after which the patient felt as bad as ever. In another the relapse occurred after only a week, and was ascribed to a sudden bereavement in the family.
In concluding, I may say that treatment by nicotine failed to give any permanent cures, although the immediate results were indisputable. The treatment should therefore be repeated at frequent intervals, say, of two to three months. Nicotine had an undoubted action in reducing muscular rigidity, and this was ascribed to the paralysing effect of the drug on the prespinal reflex arc subserving plastic tone, thus throwing new light on the dual nature of muscular tone and adducing fresh evidence that one element of muscular tone is under the control of the sympathetic nervous system.

REFERENCES.

2 2 Hall, A. J. : BRITSR MedICAL JoURYAL, January 23rd, 1926, p. 127.

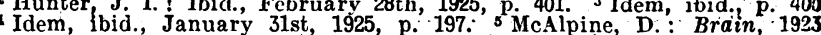
Part 3, vol. 46, p. 255. 6 Ogle, J.' W. : Medical Times and Gazette, 1804, vol. 1, p. 277 .

\section{AN EXPLANATION}

or

\section{THE TWO FORMS OF BILIRUBIN DEMONSTRATED BY THE VAN DEN BERGH REACTION.}

BY

G. A. COLIINSON, M.Sc., A.I.C., RESEARCH ASSISTANT TO SIR BERKELEY MOYNIHAN, BT., ArD

F. S. FOWWEATHER, M.D., M.So., F.I.C., D.P.H., LECTURER IN CHEMical PATHOLOG, URIVERSITY OT LEEDS; ChEMICAL PATHOLOGIST, LEEDS GENERAL INFIRMARY.

(From the Department of Pathology and Bacteriology,

To Ehrlich is due the discovery of the fact that bilirubin in acid-alcoholic solution couples with diazotized sulphanilio acid to form a dyestuff, azobilirubin. Van den Bergh has applied this reaction to the detection of bilirubin in blood serum, and hence to the clinical study of jaundice. Ho made the important observation that the bilirubin in the serum of patients suffering from obstructive jaundice reacts at once with the diazotized sulphanilic acid to give a deep red colour, while the bilirubin in serum from patients with haemolytic jaundice gives the reaction slowiy or not at all in untreated serum, but gives a fairly prompt response after treatment of the serum with alcohol and removal of the protein which is thereby precipitated. The colour obtained in the latter case is purple. With some serums an intermediate type of result is obtained-namely, the prompt development of a red colour which later becomes purple.

To interpret these results van den Bergh assumed that two forms of bilirubin exist-the one reacting directly with the sulphanilic acid reagent, and present typically in cases of obstructive jaundice, and the other reacting to an appreciable extent only in the presence of alcohol and after precipitation of protein, and present typically in cases of haemolytic jaundice. Intermediate types of result he assumed to be due to the presence of both forms.

On this assumption of two forms of bilirubin largely rests the modern theory of jaundice as described, for example, by $\mathrm{McNe} .^{1}$ The actual difference between the two forms of bilirubin which is responsible for their different behavicur has not yet been clearly demonstrated. It is considered by some that the presence of protein is responsible for the different behaviour of the two forms, the bilirubin of haemolytic jaundice being thought to be in combination with protein, while in obstructive jaundice it is in combination with an alkali. Others have attributed the different properties of the two forms to physical differences between them. It seems to us to be of considerable importance to ascertain if possible the actual mechanism underlying these different reactions, since such a knowledge must necessarily help in explaining the production of bile pigment in the body and the production of jaundice. It is with this object in view that the work about to be described has been undertaken.

It appears to us that the most important fact bearing on the problem is that bilirubin is an acid, having, according to the formula suggested by $H$. Fischer, two carboxyl groups. It is therefore capable of forming salts which 
will differ in certain properties from the free acid. This fact is already well recognized, and it is well known that bilirubin is frequently present in gall stones, principally in the form of the calciam salt, while it is generally believed that in animal fluids the pigment is present in combination with an alkali, the particular alkali not being specified.

The view we wish to put forward, together with certain experimental evidence in support, is that while the bilirubin giving the prompt direct van den Bergh reaction is an alkali salt, which we believe is probably the ammonium salt, the form which is responsible for the indirect reaction (that is, in presence of alcohol and after precipitation of protein) is the free acid. We propose first to discuss the experimental evidence in support of this view, and then to discuss its implications.

The experimental evidence may be divided into two parts-namely: (1) Comparison of the properties of free bilirubin and its salts. (2) Experiments on body fluids (bile and blood serum) containing bile pigment.

\section{Comparison of the Properties of Free Bilirubin and its Salts.}

For this purpose a quantity of free bilirubin was isolated from heavily pigmented gall stones.

The first important differences between the free acid and the alkali salts are those of solubility. Free bilirubin is easily soluble in chloroform, somewhat less soluble in alcohol, and insoluble in water; while the alkali salts are insoluble in chloroform and in alcohol, and readily soluble in water. They are moderately soluble, however, in alcohol of the strength used in the indirect van den Bergh reaction in which $2 \mathrm{c.cm}$. of 95 per cent. alcohol is added to $1 \mathrm{c.cm}$. of blood serum (that is, approximately 60 per cent. alcohol). The next important differences are in behaviour towards the van den Bergh reagent. In aqueous suspension free bilirubin only shows a colour development after standing in contact with the reagent for about twenty-four hours. The colour is of the purplish hue associated with the indirect reaction. When the free bilirubin is in-solution in 60 per cent. alcohol (forming a "true" solution) colour development occurs readily, the colour again being purple. On the other hand, the aqueous solutions of the alkali salts of bilirubin react very promptly with the van cen Bergh reagent, giving a teep ruby colour. In the case of aqueous suspensions of free bilirubin it is reasonable to assume that, with decreasing size of particles and increasing uniformity, the very long interval required before colour development occurs will be progressively shortened, the shortest interval occurring when the bilirubin is in colloidal suspension, though this is not likely to approach the rapidity with which the reaction would ocenr if the bilirubin could be obtained in true solution. As will be shown below, free bilirubin is in a colloidal state when present in blood serum, this accounting for the "delayed direct" and "biphasic" results on certain serums.

From a consideration of these chemical properties it will be seen, therefore, that with free bilirubin and its alkali salts all the results of the van den Bergh reaction can be very chosely imitated.

\section{Experiments on Body Fluids containing Bile} Pigment.

(a) The pigment of fresh bile, and of the sernms of obstructive jaundice, passes readily through a parchment membrane when subjected to dialysis, whereas the pigment of the serums of haemolytic jaundice does not pass through such a membrane unless alcohol is added. These differencès in diffusibility have already been noted by Hoover and Blankenhorn ${ }^{2}$ with respect to collodion membranes, and confirmed by others. Brulé, Garban, and Weissman ${ }^{3}$ correlated these differences with the promptness of the diazo-reaction. These results show that the pigment of bile and obstrnctive serums is in true solution, and we have found the same to be true of the alkali salts of bilirubin, while the pigment of haemolytic serums is in colloidal suspension.

(b) Both bile and obstructive serums, which normally give the prompt direct reaction, can be made to give the Indirect reaction only, if acidified with hydrochloric acid (which liberates the free bilirubin from its salts) befory adding the ran den Bergh reagent. The addition of hydrochloric acid to bile and obstructive serums also renders the pigment, originally insoluble in chloroform, soluble and extractable in that medium, thus having the properties of the free acid, while the reverse is brought about by the addition of ammonia to haemolytic serums.

The evidence in favour of the salt being that of ammonium and not one of the metals rests upon the following facts :

1. By using a technique similar to that of Nash and Benedict ${ }^{4}$ for the determination of ammonia in blood, the presence of ammonia can be demonstrated in bile, and in greater quantity than is found in blood.

2. The sodium and potassium salts of bilirubin being combinations of strong bases with a weak acid are dissociated in water to a very considerable degree, and the solutions have a markedly alkaline reaction. In attempting to convert an indirect van den Bergh reaction to a direct reaction by the addition of a caustic alkali unsatisfactory results are obtained unless the alkalinity is of a degree which cannot be conceived of as ever being present in the body fluids concerned.

3. If bile or an obstructive serum is heated, it fails to give the direct reaction, the pigment having been rendered insoluble in.water, and soluble in chloroform, by the heating process. The same is found to be true of solutions of the ammonium salt of bilirubin, heating evidently resnlting in the decomposition of the ammonium salt by loss of ammonia and consequent production of the insoluble bilirubin. This change does not occur when the metallio salts of bilirubin are similarly treated.

It will be seen, therefore, that all the experiments attempted completely confirm the view we have put forward as to the chemical natare of the two forms of bilirubin. Further support of this view, in so far as it postulates a form of bilirubin in bile which must be in true solution, is to be found in the work of Graham on cholecystography. He showed that any dyestuff which is eliminated in the bile with any degree of rapidity must be a crystalloid. Rowntree in his work on liver function tests depending on excretion of dyes into the gall bladder reaches the same conclusion. It would appear from these findings that the liver cells are only capable of allowing to pass into the bile, with any appreciable facility, such bodies as are in true solution in the blood stream.

There remains to be considered the relation of the riew put forward to the modern theory of jaundice. According to the riew now rery generally held bilirubin of the " haemolytic" form is produced by the cells of the reticuloendothelial system, in the liver and elsewhere. In its passage through the liver cells to the bile capillaries it becones converted to the "obstructive" form in which it is found in the bile. Applying our views to this theory it follows that the bilirubin produced by the cells of the reticulo-endothelial system is in the form of free bilirubin, and the site of formation of the ammonium salt is in the liver cells.

The probability of this site of formation of the ammonium salt is supported by various considerations. In the first place a condition of alkalinity is required. That such a condition may be found in the liver cells is suggested by work on other subjects; thus Wells, ${ }^{5}$ discussing certain aspects of sugar metabolism, says: "It might be assumed that all sugars upon entering certain phases of the cells (phases especially well represented in liver cells) meet conditions which are equivalent to those met in weakly alkaline solutions. . . That is, especially in the liver there may be the equivalent of dilute alkali for all sugars."

Apart from these speculations, many observers are agreed that the pigment of bile, of whose origin in the liver there is no doubt, is in the form of "bilirubinalkali."

In the second place there must be a source of ammonia. This is probably to be found as a result of the functions of deamination of amino-acids and formation of urea carried on by the liver cells. Though the actual mechanism of the procluction of urea from amino-acids is still a matter of speculation it is probable that ammonia is the immediate, if short-lived, precursor of urea. The presence of 
bilirubin at the site of production of ammonia, though the latter may exist as such for only a very short time, is sufficient to explain the formation of the bilirubin ammonium salt. The liver cells are, in fact, the most probable site of formation of the ammonium salt.

Our views, therefore, as to the chemical nature of the two forms of bilirubin do not in any way conflict with the modern theory of jaundice, but on the other hand appear to lend it some support.

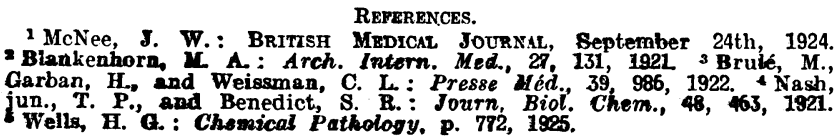

\section{DOUBLE VAGINA AND SINGLE UTERUS. sY}

R. C. BARTLETT, M.R.C.S., L.R.C.P., ROMBEY, HAMTS.

Is the Jounnal of March 13th (p. 476) Dr. Macgown of Kong-Kong reports a remarkable and interesting case of double uterus with double vagina, in which, occasion for Caesarean section having arisen, he was given the opportunity of verifying his previous clinical observations as to the degree of duplication of the uterus.

In the rare lapses on the part of Nature from her usual wonderful precision the departure from normal in failure to effect fusion of the Mullerian ducts increases as the failure extends from the level of the Fallopian tubes downwards. Thus aterus didelphys (or uterus septus) with double vagina, as met with by Dr. Macgown, is an exceedingly rare condition. A reversal in the order of fusion by which double vagina is associated with complete coalescence in the body of the uterus and the uterine cavity is probably the rarest of all such abnormalities. In the limited field of research at my immediate disposal I find no such case recorded, but that a few cases similar to the one I now wish to describe can be cited $I$ do not doubt.

A woman, aged 29, married seven years, nulliparous and desirous of children, consulted me solely in respect of the unfertility of her marriage. She was of good physique and health, menstruation had been regular, and she was aware of no sexual incapacity or anatomical defect.

On digital examination the ostium vaginae and the vagina itself appeared normal, and the cervix uteri was fully developed and centrally situated, but, as it seemed, a thin membrane covered the whole of the vaginal cervix, intervening between it and the examining finger. On preparing to insert a vaginal speculum vaginae, and tucked away against the left labium, what at first sight looked like a second meatus urinarius. The meatus urethrae proper was in its natural position anteriorly. On drawing the labium further aside there was displayed an intact left hymen hasing a natural opening no larger than would easily admit a No. 10 catheter. The next day, under a general angesthetic, No broke down this left hymen and discovered a completely separate left vagina with a normal cervix and cervical canal beyond it. This was, of course, the cervix $I$ had previously felt through the septum from the right vagina. Right up in the fornix of the right vagina was $a$ rudimentary bud-like right vaginal cervix no larger than a hazel-nut. It had an umbilicated central depression but no patent cervical canal, and it appeared to spring from the side of the left (main) cervix at the level of the internal os. The body of the uterus, as far as I could determine its outline bimanually, was neither bicornuate nor unicornuate. The raginal septum was thick and fleshy below, thin and membranous above, and it extended the whole length of the vagina right up to the angle formed by the rudimentary right cervix. Either vagina admitted a $1 \frac{1}{4}$-inch Fergusson's speculum easily, the septum being deflected to the opposite side. The two blades of a bivalve speculum, being passed, one into each vagina, displayed the septum and its attachments perfectly.

Evidently the menstrual flow had always been through the left vagina, and probably the virginal condition of the right hymen had been similar to that of the left. Had the left hymen given way instead of the right on the patient's marriage, I could see no reason to doubt that impregnation would have occurred, or to suppose that the vaginal septum would have opposed any resistance in parturition, and so have been destroyed. I think it even possible that the very existence of the right vagina might then have been unnoticed in the ordinary course of obstetric attendance. However that may have been, in deference to the patient's paramount desire to have children, and to give her the greater chance of its fulfilment, I decided to throw the two vaginae into one by division or excision of the septum. Accordingly the septum was divided by scissors from below upwards, and the cut edges of the lower thick parts were sewn over by a few loops of continuous suture. The flaps retracted and shrivelled, and when I next saw the patient, eight months later, little more than an anterior and a posterior raphé remained. She was then already five months pregnant. Gestation was perfectly normal throughout and went to its full term, and in due course $I$ delivered her of a well developed male child after a rather easy labour. She subsequently, as I was informed, bore another (female) child-again without difficulty.

Apart from the fact that neither before nor during pregnancy could I detect a separate right uterine body, or any vestige thereof (although therein I may have been mistaken), my reasons for believing that only the vagina was double are: (1) that there was no history of haematometra or other pelvic crisis arising from puberty, and (2) that the raginal septum, while thick and fleshy below, thinned almost to the vanishing point at the fornix. Against such view may be urged the existence of a rudimentary right cervix. But this appeared to bo integumentary rather than substantial in structure, and, that being so, would it not morphologically belong to the vagina rather than to the uterus?

\section{Atemoranta: MEDICAL, SURGICAL, OBSTETRICAL.}

\section{TUBERCULIN LINIMENT.}

ThE value of tuberculin in the treatment of tuberculosis is enhanced if one realizes the conditions under which the best results are likely to be obtained. Some two years ago, as the result of an analysis of 96 cases treated with tuberculin, I formed a working theory as to the class of case in which it was likely to prove beneficial. The details were published in the Journal. ${ }^{1}$ I have had no reason to alter the conclusions to which $\mathbf{I}$ then came, which were:

1. That fibrosis is an important factor in tuberculin therapy, and that other things being equal, the less fibrosis there be the more satisfactory will be the results with tuberculin.

2. That liypersensitiveness is no contraindication to tuberculin therapy.

3. That in subsensitive cases much improvement cannot be expected except in asthma.

By far the best results, in my experience, are obtained in hypersensitive cases and in asthma. The difficulty is that if hypersensitive subjects be treated with hypodermic injections of tuberculin the general reactions may be unduly serere, and in the asthmatics an attack of asthma may be precipitated. For these reasons one is apt to be deterred from giving tuberculin in the rery cases in which it is such a powerful agent for good. Fortunately, there are other ways of exhibiting tuberculin besides the hypodermic method.

Sir Robert Philip has advocated ${ }^{2}$ the administration of tuberculin as an ointment. I use this method frequently with satisfactory results. It has, howerer, the disadrantage that the dasage cannot be accurately regulated. Dr. James Crocket ${ }^{3}$ speaks favourably of tuberculin liniment. I have given this method an extensive trial and have found it valuable. A description of the procedure adopted may therefore be of interest.

The patient is first tested as to sensitiveness to tuberculin by the von Pirquet method modified by . Ellis. This consists in performing a cutaneous test with dilutions of ald classified as hypersensitive, those to 1 in 100 as sensitive, and those to 1 in 10 as subsensitive. A skiagram of the lungs is taken and the extent of fibrosis, if present, is noted. The test is essential and the of fibrosis, if present, is noted. as indicated above, a knowledge $x$-ray report most desirable, for, as indicated above, a knowledge of the degree of sensitiveness to tuberculin and the amount of
fibrosis is the keynote to successful tuberculin therapy and to prognosis.

The patients take their own temperatures four times a day and their weight is recorded weekly. They attend hospital once or twice a week for treatment, which is conducted as follows: $0.01 \mathrm{c.cm}$. of T.A.F. (" tuberculin albumose frei ") is diluted with a suitable quantity (say 0.5 c.cm.) of compound camphor liniment. This the patient rubs into the back of his upper arm. If there be no local reaction to this the dose is doubled at the next sitting, and doubling is continued at each subsequent sitting till

1 BRITISH MEDICAL JOURNaL, Junc 14th, 1924

2 Ibdisburgh Medical Journal, March, 1924. 